\title{
Emergence of Writing Systems through Multi-Agent Cooperation (Student Abstract)
}

\author{
Shresth Verma, Joydip Dhar \\ ABV-Indian Institute of Information Technology and Management \\ Gwalior, MP, India 474003 \\ \{vermashresth, jdhar.iiitmg\}@gmail.com
}

\begin{abstract}
Learning to communicate is considered an essential task to develop a general AI. While recent literature in language evolution has studied emergent language through discrete or continuous message symbols, there has been little work in the emergence of writing systems in artificial agents. In this paper, we present a referential game setup with two agents, where the mode of communication is a written language system that emerges during the play. We show that the agents can learn to coordinate successfully using this mode of communication. Further, we study how the game rules affect the writing system taxonomy by proposing a consistency metric.
\end{abstract}

\section{Introduction}

Recent advances in deep learning have shown exceptional results in language-related tasks such as machine translation, question answering, or sentiment analysis. However, the supervised approaches that capture the underlying statistical patterns in language are not sufficient in perceiving the interactive nature of communication. It is thus crucial to learn to communicate by interaction, i.e., communication must emerge out of necessity.

Several recent works (Lazaridou, Peysakhovich, and Baroni 2017; Lazaridou et al. 2018; Mordatch and Abbeel 2018), have shown that in multi-agent cooperative setting of referential games, deep reinforcement learning can successfully induce communication protocols. In such games, communication success is the only supervision during learning, and the meaning of the emergent messages gets grounded during the game. In (Lazaridou, Peysakhovich, and Baroni 2017), the authors have restricted the message to be a single symbol token picked from a fixed vocabulary while in (Lazaridou et al. 2018), the message is considered to be a sequence of symbols. The latter work also demonstrates successful communication in environments with raw perceptual inputs. (Mordatch and Abbeel 2018), further extends the scope of the mode of communication by including the emergence of non-verbal communication in their work. While all of these works have studied a wide variety of game setups as well as variations in communication rules, none of

Copyright (c) 2020, Association for the Advancement of Artificial Intelligence (www.aaai.org). All rights reserved. them have considered written language system as a mode of communication. Historically, written language systems have shown complex patterns in evolution over time. Moreover, the process of writing requires sophisticated graphomotor skills which involves both linguistic and non-linguistic factors. Thus writing systems can be considered crucial for understanding autonomous system development. We are further motivated by the work in (Ganin et al. 2018), where the authors demonstrate that artificial agents can produce visual representations similar to those created by humans. We extend this idea to study the emergence of writing systems.

\section{Referential Game Framework}

There are two players, a sender and a receiver. From a given set of images $I=\left\{i_{j}\right\}_{j=1}^{N}$, we sample a target image $t \in I$ and $K-1$ distracting images $D=\left\{d_{j}\right\}_{j=1}^{K-1}, d_{j} \in I$ s.t. $\forall j t \neq d_{j}$. Now, we define two sender types, Distractor Agnostic (D-Agnostic): where the sender only has access to the target image $t$; Distractor Aware (D-Aware): where the sender has access to the candidate set $C=t \cup D$. In both these variations, the sender has to come up with a message $M_{l}=\left\{m_{j}\right\}_{j=1}^{l}$, which is a sequence of $l$ brushstrokes. A black-box renderer $\mathcal{R}$ accepts the sequence of brushstrokes $M_{l}$ and paints them onto a canvas. This results in a written symbol image $W=\mathcal{R}\left(M_{l}\right)$. Given the written symbol image $W$ and the candidate set $\mathrm{C}$, the receiver has to identify the target image $t$. Communicative success is achieved when the target is correctly identified, and a payoff of 1 is assigned to both the players. In rest of the cases, the payoff is 0 .

\section{Experimental Setup}

\section{Agents}

The sender and receiver are modelled as reinforcement learning policy networks $S_{\theta}$ and $R_{\phi}$. Specifically, the sender is a recurrent neural network which takes as input the current state of the canvas along with the visual input $V$ which can either be target image $t$ (D-Agnostic) or candidate set $C$ (D-Aware). At the $i^{\text {th }}$ timestep, the sender outputs a brushstroke $m_{i}$. The canvas state is the intermediate rendering $\mathcal{R}\left(M_{i}\right)$, where $M_{i}$ is the collection of brushstrokes produced up to timestep $i$. Thus, $m_{i+1}$ is generated by sampling 
from $S_{\theta}\left(\mathcal{R}\left(M_{i}\right), h_{i}, V\right)$ where $h_{i}$ is the internal hidden state maintained across timesteps. The sequence is terminated when either the maximum sequence length $L$ is reached, or a terminal flag is produced along with the brushstroke. The receiver agent first extracts features from the written symbol image $W$. For creating brushstrokes that are similar to written languages used by humans, we use feature extractor from a Siamese Neural Network pre-trained on the OMNIGLOT dataset (Koch, Zemel, and Salakhutdinov 2015). Given the written symbol image $W$, a candidate set $\mathrm{U}$ (a random permutation of $\mathrm{C}$ ), and the feature extractor $f_{s}$, the receiver returns an integer value $t^{\prime}=R_{\phi}\left(f_{s}(W), U\right)$ in the range 0 to $\mathrm{K}-1$ that points to the target.

\section{Learning}

For both the agents, we pose the learning of communication protocols as maximization of the expected return $E_{\tilde{r}}[R(\tilde{r})]$, where $R$ is the reward function. The payoff is 1 for both the agents iff $R_{\phi}\left(f_{s}\left(S_{\theta}\left(\mathcal{R}\left(M_{i}\right), h_{i}, V\right)\right), U\right)=t$, where $i$ is the last timestep of the episode. In all other cases and intermediate timesteps, the payoff is 0 . Because of the high dimensional search space introduced due to brushstrokes, we use Proximal Policy Optimization (PPO) (Schulman et al. 2017) for optimizing the weights of sender and receiver agents.

\section{Images}

We have used CIFAR-10 dataset (Krizhevsky, Hinton, and others 2009), as a source of images. From the test set of CIFAR-10, we randomly sample 100 images from each class and represent them as outputs from relu7 layer of pretrained VGG-16 convNet (Simonyan and Zisserman 2015).

\section{Results and Conclusion}

Figure 1 shows the performance of our game setup for both the sender variations. The agents converge to coordination in both sender types, but D-Aware sender reaches higher levels more quickly. Further, we quantify the consistency of a writing system by studying the variability of the symbols produced for a given entity $e$. Let $w_{e}$ be the set of all written symbol images representing $e$. We define heatmap $H_{e}=\operatorname{mean}\left(w_{e}\right)$. For a writing system consistent for the entity $e, H_{e}$ would contain sharp brushstrokes while a nonconsistent writing system would give a blurred heatmap. We thus compute the Variance of Laplacian (VoL) of the heatmap to quantify sharpness. Table 1 reports the average consistency score given by $\frac{\sum_{e \in E} V o L\left(H_{e}\right)}{|E|}$ where $E$ is the set of all the entities considered which can either be targets $(t)$ or all possible target-distractor combinations $(t \& d)$. Note that $(t \& d)$ configuration is not possible in D-Agnostic sender. We also report a baseline consistency score for comparison where the heatmap is generated by averaging across the universal set of generated symbol images.

High consistency of D-Agnostic sender indicates a oneto-one mapping from target class to written symbols. The D-Aware sender has low consistency over target class but high consistency for target-distractor combinations. This means that target symbols are distractor-dependent. From

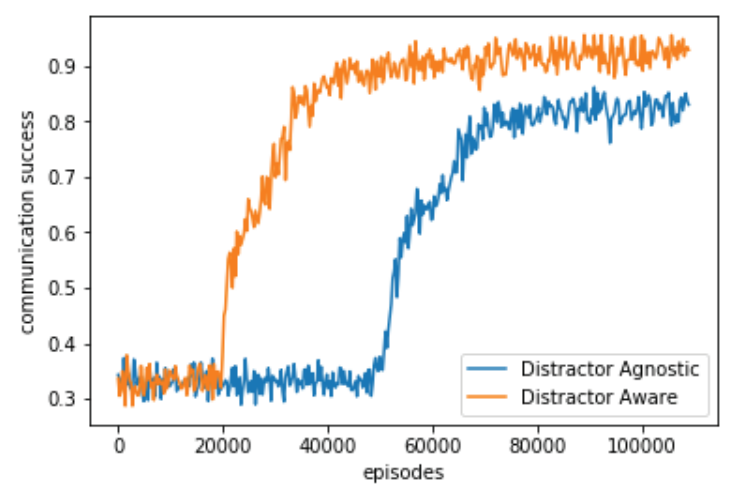

Figure 1: Communication success as a function of training episodes for referential games with $\mathrm{K}=3$ and $\mathrm{L}=2$

\begin{tabular}{|c|c|c|}
\hline Sender Type & $\begin{array}{l}\text { Average Consistency } \\
\text { Score }\end{array}$ & $\begin{array}{l}\text { Baseline Consistency } \\
\text { Score }\end{array}$ \\
\hline D-Agnostic $^{t}$ & 0.019 & 0.0055 \\
\hline D-Aware ${ }^{t}$ & 0.007 & 0.0044 \\
\hline D-Aware ${ }^{t \& d}$ & 0.015 & 0.0044 \\
\hline
\end{tabular}

Table 1: Consistency scores for different sender types

our qualitative evaluations, we infer that D-Aware sender assigns meaning to brushstrokes that represent conceptual differences between target and distractors. Furthermore, DAgnostic sender uses a scheme akin to hierarchical encoding to attribute high-level semantics to brushstrokes. Thus, the writing system emerging from D-Aware sender is an ideographic one representing concepts while D-Agnostic sender produces a writing system which has compositionality and shows logographic traits.

\section{References}

Ganin, Y.; Kulkarni, T.; Babuschkin, I.; Eslami, S. M. A.; and Vinyals, O. 2018. Synthesizing programs for images using reinforced adversarial learning. In ICML, volume 80 of Proceedings of Machine Learning Research, 1652-1661. PMLR.

Koch, G.; Zemel, R.; and Salakhutdinov, R. 2015. Siamese neural networks for one-shot image recognition. In Proc. ICML deep learning workshop, volume 2.

Krizhevsky, A.; Hinton, G.; et al. 2009. Learning multiple layers of features from tiny images. Technical report, Citeseer.

Lazaridou, A.; Hermann, K. M.; Tuyls, K.; and Clark, S. 2018. Emergence of linguistic communication from referential games with symbolic and pixel input. In ICLR. OpenReview.net.

Lazaridou, A.; Peysakhovich, A.; and Baroni, M. 2017. Multiagent cooperation and the emergence of (natural) language. In ICLR. OpenReview.net.

Mordatch, I., and Abbeel, P. 2018. Emergence of grounded compositional language in multi-agent populations. In AAAI, 1495-1502. AAAI Press.

Schulman, J.; Wolski, F.; Dhariwal, P.; Radford, A.; and Klimov, O. 2017. Proximal policy optimization algorithms. CoRR abs/1707.06347.

Simonyan, K., and Zisserman, A. 2015. Very deep convolutional networks for large-scale image recognition. In ICLR. 